\title{
Effect of Microelements and Selenium on Superoxide Dismutase Enzyme, Malondialdehyde Activity and Grain Yield Maize (Zea mays L.) under Water Deficit Stress
}

\author{
Nourali SAJEDI ${ }^{1}$, Hamid MADANI $^{1}$, Ahmad NADERI ${ }^{2}$ \\ ${ }^{1}$ Islamic Azad University, Department of Agronomy and Plant Breeding, Arak Branch, Arak, Iran; n-sajedi@iau-arak.ac.ir (coressponding author) \\ ${ }^{2}$ Research Center of Agriculture and Natural Source of Khuzestan, Iran; Ah_naderi36@yahoo.com
}

\begin{abstract}
This study was carried out to investigate effects of microelements under water deficit stress at different growth stages on antioxidant enzyme alteration, chemical biomarker and grain yield of maize in the years 2007 and 2008. The experiment was conducted in a split plot factorial based on a randomized complete block design with four replications. There were three factors, water deficit stress at different stages of growth as main plot and combinations of selenium (with and without using) and microelements (with and without using) as sub plots. The result indicated that the activity of superoxide dismutase and malondialdehyde content under water deficit stress increased, but grain yield was reduced. The highest grain yield was obtained from optimum irrigation, while in the case of with water deficit stress at $\mathrm{V}_{8}$ stage it was non significant. Selenium spray increased activity of superoxide dismutase enzyme, malondialdehyde content of leaves in $\mathrm{V}_{8}, \mathrm{R}_{2}$ and $\mathrm{R}_{4}$ stages and also grain yield. Application of microelements increased the leaves superoxide dismutase enzyme activity and malondialdehyde content. Selenium and microelements spray under water deficit stress conditions during vegetative growth and dough stage increased grain yield in comparison to not spraying elements under water stress conditions. The present results also showed that by using selenium and microelements under water stress can obtain acceptable yield compared to not using these elements.
\end{abstract}

Keywords: maize, microelements, selenium, superoxide dismutase enzyme, malondialdehyde

\section{Introduction}

Maize (Zea mays L.) is the world's most widely grown cereal and is the primary staple food in many developing countries (Morris et al., 1999). About 26\% of the world's total cultivable land falls in arid and semi-arid areas (Paylore and Greenwell, 1979). The exposure of plants to environmental stresses such as drought, heat, chilling, salt and plant diseases can result in the production of reactive oxygen species (ROS) that contributes to diminished plant performance (Cheong et al., 2003). These abiotic stresses can result in the accumulation of reactive oxygen species (ROS) and other toxic compounds (Xiong et al., 2002). Production of ROS during environmental stress is one of the main causes for decreases in productivity, injury, and death that accompany these stresses in plants. ROS are produced in both unstressed and stressed cells, and in various locations (Upadhyaya and Panda, 2004). In plant cell chloroplasts, mitochondria and peroxisomes, there are important intracellular generators of ROS (Elstner, 1991). ROS play an important role in endonuclease activation and consequent DNA damage (Hagar et al., 1996). Oxidative stress can prevent photosynthetic activity, respiration process and plant growth. Plants are naturally provided by enzymatic and non-enzymatic systems to take care of active oxygen (Giang and Huang, 2001). Photosynthetic plants have a strong demand for combating oxidative stress and other abiotic stresses (Xiong et al., 2002). Plant cells respond defensively to oxidative stress by removing the ROS and maintaining antioxidant defense compounds at levels that reflect ambient environmental conditions (Scandalios, 1997). The mechanisms that act to adjust antioxidant levels afford the protection and include changes in antioxidant gene expression (Cushman et al., 2000). Some well-known antioxidants in plants include glutathione, vitamin $\mathrm{C}$, vitamin $\mathrm{E}$, antioxidant enzymes and carotenoids. Catalases, superoxide dismutase, peroxides, are antioxidant enzymes. Bailly et al. (2000) reported that the content of superoxide dismutase (SOD), catalase (CAT), glutathione reductase (GR) and malondialdehyde (MDA) in sunflower seeds will increase under drought stress condition. Within a cell, superoxide dismutase (SOD) constitutes the first line of defense against ROS (Alscher et al., 2002). Malondialdehyde has been known as the end product of peroxidation of membrane lipids. Water deficit stress by increase of generation ROS is responsible for stress-dependent peroxidation of membrane lipids (Upadhyaya and Panda, 2004).

Searching for suitable ameliorants or stress alleviant is one of the tasks of plant biologists. Recent researches 
154

have identified several beneficial effects of selenium (Se) in plants although Se is not considered to be required by higher plants. Positive effects of Se on plants mainly exhibited: promoting plant growth, alleviating UV-induced (Yao et al., 2009). Selenium is an element whose deficiency causes the decrease in defense mechanisms of living organisms. Earlier studies have indicated that selenium (Se) maintains antioxidative defence systems and enhances sugar and starch accumulation. Among naturally existing elements seven micro elements including i.e., $\mathrm{Fe}, \mathrm{Zn}, \mathrm{Cu}$, $\mathrm{Mn}, \mathrm{B}, \mathrm{Mo}$ and chlorine are needed for plant growth. Ionic proms of $\mathrm{Fe}, \mathrm{Zn}, \mathrm{Cu}, \mathrm{Mn}$ and $\mathrm{Mg}$ as co-factors exist in many antioxidant enzymes. Under deficiency of microelementss antioxidant enzymes activity is reduced, therefore plants sensitivity to environmental stresses increases (Cakmak, 2000). Application of microelement fertilizers can enhance plants resistance to environmental stresses such as drought and salinity (Maleckuti and Sepehri, 2001; Movahed Dehnaviet et al., 2002). Malan et al (1990) reported that drought-tolerant and intolerant maize inbred correlated with $\mathrm{Cu} / \mathrm{Zn} \mathrm{SOD}$ and glutathione reductase activities although higher levels of one enzyme alone apparently did not confer drought tolerance. The relative tolerance of a genotype to water stress as reflected by its comparatively lower lipid peroxidation and higher membrane stability index, chlorophyll and carotenoid contents was closely associated with its antioxidant enzyme system (SOD, APO, GR, CAT). Results of experiments indicated that micronutrient application reduces the effects of environmental stresses such as drought stress and salt stress (Wang et al., 2004).

The objective of this research was to investigate the effects of selenium and microelements spraying under deficit stress on maize yield and biochemical characteristics at different growth stages.

\section{Materials and methods}

This study was conducted under water deficit stress with maize (Zea mays L. 'S.C 704'). These experiments were carried out in the Agricultural Research Station of Islamic Azad University, Arak Branch, Iran, during the growing season of 2007-2008 and 2008-2009. This site is located at $34^{\circ} 30^{\prime} \mathrm{N}$ latitude, $40^{\circ} 41^{\prime} \mathrm{E}$ longitude, with the altitude of $1779 \mathrm{~m}$ above sea level in Markazi Province in the center of Iran. This region has a semi-arid climate on the base of metrological data in Arak, Iran in 2007-2008 and 2008-2009 (Tab. 1).

Experimental treatments were irrigation levels in the main plots including full watering as control, water stress in vegetative stage $\left(\mathrm{V}_{8}\right)$, seed formation or blister stage $\left(R_{2}\right)$ and grain pre maturity or dough stage $\left(R_{4}\right)$. The sub plots included mix levels of selenium and microelements fertilizer. Water stress was executed by temporary stopping of irrigation at each mentioned stage. Full irrigation (control) was arranged by crop water requirement according to
Tab. 1. Metrological data in Arak, Iran

\begin{tabular}{ccccc}
\hline \multirow{2}{*}{ Month } & \multicolumn{2}{c}{$\begin{array}{c}\text { Monthly total of } \\
\text { precipitation }(\mathrm{mm})\end{array}$} & \multicolumn{2}{c}{$\begin{array}{c}\text { Average of } \\
\text { temperature }\left({ }^{\circ} \mathrm{C}\right)\end{array}$} \\
\cline { 2 - 5 } & $2007-2008$ & $2008-2009$ & $2007-2008$ & $2008-2009$ \\
\hline May & 17.1 & 17.9 & 16.4 & 19.5 \\
June & 23.7 & 23.4 & 5.4 & 0 \\
July & 27.1 & 27.7 & 36.5 & 0.1 \\
Aug & 26.2 & 27.1 & 7.3 & 0 \\
Sep & 23.7 & 23.8 & 0.1 & 0 \\
Oct & 17.6 & 16.9 & 1.5 & 15 \\
Mean & 22.5 & 22.8 & 18.7 & 5.8 \\
\hline
\end{tabular}

daily evaporation from basin pan. Daily evaporation from basin pan was calculated by equation of

$\mathrm{V}=\mathbf{S} \times \mathbf{H}$

$\mathrm{V}$ : Daily evaporation from basin pan

$S$ : Area of basin pan

$\mathrm{H}$ : Rate of evaporated water

Also from multiple coefficients of basin pan and evaporated water, the potential of evapotranspiration was obtained. Rate of entered water to every plot was calculated by the following equation (Alizadeh, 2002).

Water volume $\left(\mathbf{m}^{3}\right)=$ Plot area $\times$ Irrigation water efficiency $\times$ Maize coefficient $\times$ Potential of evapotranspiration

Maize coefficient is 0.36 up to 0.58 at growth initiation, 0.71 up to 1.13 at growth meddle and 0.98 up to 0.68 at growth final (Farshi et al., 1997). Irrigation water efficiency of $80 \%$ was considered. Irrigation type was a siphoning system with polyethylene tubes that was controlled by tube regulator tap. Total of consumptive water during season of corn for every treatment was calculated (Tab. 2).

Microelements fertilizer (Biomin 212) treatments were in two levels without and with application. Foliar application at early vegetative stage $(\mathrm{V})$ and a week before tassling stage at the rate of 2 liter ha ${ }^{-1}$. Biomin 212 fertilizer content (\%wt/wt) was $2.0 \mathrm{Fe}, 2.0 \mathrm{Zn}, 0.5 \mathrm{Cu}, 1.0 \mathrm{Mn}$, $0.025 \mathrm{~B}$ and $0.5 \mathrm{Mg}$.

Selenium treatments were at two levels, as well. The first one was selenium application with $20 \mathrm{~g} \mathrm{ha}^{-1}$ sodium selenite at early vegetative stage $\left(\mathrm{V}_{6}\right)$ and second one was control.

Tab. 2. Volume of consumptive water

\begin{tabular}{ccc}
\hline Year & Experiments of irrigation & $\begin{array}{c}\text { Irrigation } \\
\text { water }\left(\mathrm{m}^{3} / \mathrm{ha}\right)\end{array}$ \\
\hline \multirow{2}{*}{$2007-2008$} & optimum condition & 8552 \\
& water deficit stress in $\mathrm{V}_{8}$ & 7205 \\
& water deficit stress in $\mathrm{R}_{2}$ & 7295 \\
& water deficit stress in $\mathrm{R}_{4}$ & 7278 \\
\hline \multirow{2}{*}{$2008-2009$} & optimum condition & 7939 \\
& water deficit stress inV & 7871 \\
& water deficit stress in $\mathrm{R}_{2}$ & 7904 \\
& water deficit stress in $\mathrm{R}_{4}$ & 7847 \\
\hline
\end{tabular}


Tab. 3. Chemical and physical properties of farm soil

\begin{tabular}{cccccccccccccccc}
\hline Year & Depth & EC & $\mathrm{pH}$ & $\mathrm{OC}$ & $\mathrm{N}$ & $\mathrm{P}$ & $\mathrm{K}$ & $\mathrm{Zn}$ & $\mathrm{Fe}$ & $\mathrm{Mn}$ & $\mathrm{Cu}$ & $\mathrm{Se}$ & Sand & Silt & Clay \\
\hline & $(\mathrm{cm})$ & $\left(\mathrm{dsm}^{-1}\right)$ & \multicolumn{6}{c}{$(\%)$} & & \multicolumn{7}{c}{$(\mathrm{ppm})$} & & & & & $(\%)$ & \\
2007 & $0-30$ & 1.20 & 7.5 & 0.82 & 0.08 & 5 & 150 & 0.8 & 4.6 & 10.6 & 1.14 & 0.27 & 29 & 35 & 36 \\
2008 & $0-30$ & 1.70 & 7.7 & 0.78 & 0.06 & 8.5 & 130 & 9.2 & 3.9 & 9.2 & 1.1 & 0.23 & 26 & 38 & 36 \\
\hline
\end{tabular}

Before seed sowing, multiple soil samples were collected for determination of their physical and chemical properties (Tab. 3). According to soil testing the amounts of fertilization were applied including $375 \mathrm{kgha}^{-1}$ urea, $150 \mathrm{kgha}^{-1}$ triple super phosphates and $150 \mathrm{kgha}^{-1}$ potassium sulphate fertilizers. 30 percentages of nitrogen $(\mathrm{N})$ and all of phosphorous $(\mathrm{P})$ and potassium $(\mathrm{K})$ fertilizers were applied at planting time. The remaining $\mathrm{N}$ fertilizer was broadcasted twice during the vegetative growth as top-dress fertilizer at six-leaf stage and two weeks before tassling stage.

Each experimental plot included four $60 \mathrm{~cm}$ distanced rows with $20 \mathrm{~cm}$ spacing between plants in rows. Land preparation, including ploughing, was conducted in fall and perpendicular disking in May The length of each row was $6 \mathrm{~m}$ and two rows were left uncultivated between the adjacent plots. The hybrid maize was 'S.C 704'. The seeds were sown at $50-60 \mathrm{~mm}$ depth on $18^{\text {th }}$ May, 2007(first year) and $14^{\text {th }}$ May, 2008 (second year).

Biochemical analysis was based on plant sampling $24 \mathrm{~h}$ before irrigation times from five leaves of each plant. The harvested leaves were frizzed and kept at $-80^{\circ} \mathrm{C}$ for further biochemical analysis. Leaf samples $(0.2 \mathrm{~g})$ were homogenized in a mortar and pestle with $3 \mathrm{ml}$ ice-cold extraction buffer (25 mM sodium phosphate, $\mathrm{pH} 7.8$ ). The homogenate plant material was centrifuged by $18000 \mathrm{rm}^{-1}$ for 30 minutes, and then the supernatant was filtered through filter paper. The supernatant fraction was used as a crude extract for the assay of enzyme activity. The biochemical activity was measured based on Misra and Fridorich (1979). The ability to inhibit free radical chain-propagating radi$\mathrm{cal}$ and the auto oxidation of epinephrine $(0.25 \mathrm{mM})$ were determined. Furthermore, SOD standard was used for calibration of activity and lipid peroxidation was determined by estimating the malondialdehyde (MDA) content in $1 \mathrm{~g}$ fresh leaf material according to Madhava Rao and Sresty

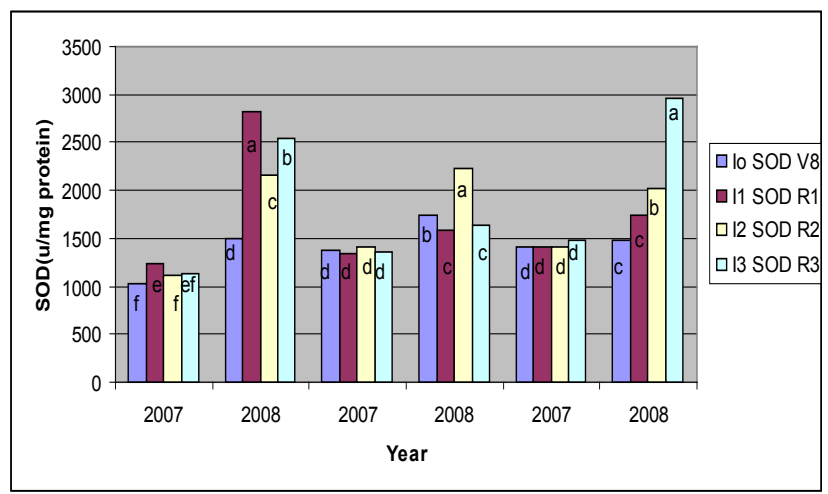

Fig. 1. Effect year on SOD enzyme activity
(2000). Determination of MDA that is a product of lipid peroxidation was determined by thiobarbituric acid reaction. The concentration of MDA was calculated from the spectroscopy absorbance at $532 \mathrm{~nm}$ (correction was done by subtracting the absorbance at $600 \mathrm{~nm}$ for unspecific turbidity).

Two years data were combined and the analysis of variance was performed using SAS (SAS Institute Inc, 1997). Each treatment was analyzed in four replications. The means comparisons were estimated by Tukey's multiple tests at $\% 5$ probability level.

\section{Results and discussion}

According to the combined mean comparison results, the years significantly affected superoxide dismutase enzyme activity and malondialdehyde content. The superoxide dismutase enzyme activity and malondialdehyde leaves content in the second year in all three plant growth and development stages were higher than in the first year (Tab. 4, Fig. 1 and 2).

In this study, the highest and lowest superoxide dismutase enzyme activity and malondialdehyde content of leaves was observed in water deficit stress for $\mathrm{V}_{8}$ and $\mathrm{R}_{4}$ stage respectively. Under water deficit condition, the ROS generation and antioxidant enzymes activity increased at $\mathrm{V}_{8}$ and $\mathrm{R}_{4}$ growth and development stages. In other words, water deficit condition in $\mathrm{V}_{8}, \mathrm{R}_{2}$ and $\mathrm{R}_{4}$ in maize growth and development stages could be increase malondialdehyde level by 21,23 and $26 \%$ respectively compared to control (Tab. 4). The simultaneous increase in these enzymes activity contributes to a decrease of injurious effects of $\mathrm{H}_{2} \mathrm{O}_{2}$ under drought stress. As plants produce new organs and have high metabolism at $\mathrm{V}_{8}$ stage, there upon ROS generation will increased under water deficit stress condition. Upadhyaya and Panda (2004) reported that

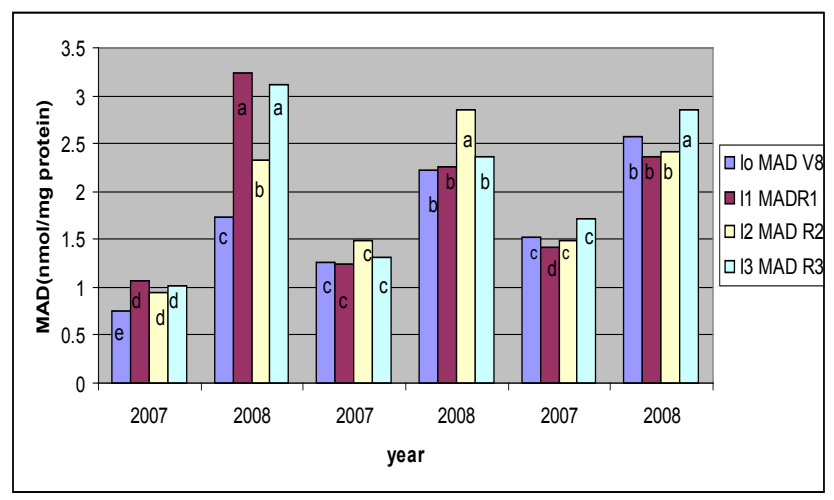

Fig. 2. Effect year on MAD content 
Tab. 4. Mean comparisons for traits at different treatment levels

\begin{tabular}{|c|c|c|c|c|c|c|c|}
\hline \multirow{2}{*}{ Treatment } & $\mathrm{SODV}_{8}$ & $\mathrm{SOD} \mathrm{R}_{2}$ & $\mathrm{SOD} \mathrm{R}_{4}$ & $\mathrm{MADV}_{8}$ & $\mathrm{MAD} \mathrm{R}_{2}$ & $\mathrm{MAD} \mathrm{R}_{4}$ & Grain yield \\
\hline & \multicolumn{3}{|c|}{ (u/mg.protein) } & \multicolumn{3}{|c|}{ (nm/mg.protein) } & $\mathrm{Kg} \mathrm{ha}^{-1}$ \\
\hline \multicolumn{8}{|l|}{ Year } \\
\hline 2007 & $1132.97 \mathrm{~b}$ & $1373.01 \mathrm{~b}$ & $1434.25 b$ & $0.95 b$ & $1.33 \mathrm{~b}$ & $1.54 \mathrm{~b}$ & $6781.55 \mathrm{a}$ \\
\hline 2008 & $2257.42 \mathrm{a}$ & $1802.57 \mathrm{a}$ & $2136.39 \mathrm{a}$ & $2.61 \mathrm{a}$ & $2.43 \mathrm{a}$ & $2.55 \mathrm{a}$ & $6118.14 a$ \\
\hline \multicolumn{8}{|c|}{ Water Limitation } \\
\hline $\mathrm{L}_{1}$ & $1263.93 \mathrm{~d}$ & $1560.50 \mathrm{~b}$ & $1575.96 \mathrm{c}$ & $1.25 \mathrm{c}$ & $1.75 b$ & $2.05 b$ & $7076.44 a$ \\
\hline $\mathrm{L}_{2}$ & $2031.25 \mathrm{a}$ & $1466.59 \mathrm{c}$ & $1627.37 \mathrm{bc}$ & $2.15 \mathrm{a}$ & $1.75 b$ & $1.89 \mathrm{~b}$ & $6603.75 \mathrm{ab}$ \\
\hline $\mathrm{L}_{3}$ & $1642.46 \mathrm{c}$ & $1825.75 a$ & $1716.93 b$ & $1.63 \mathrm{~b}$ & $2.18 \mathrm{a}$ & $1.95 b$ & $4241.05 c$ \\
\hline $\mathrm{L}_{4}$ & $1841.78 \mathrm{~b}$ & $1498.34 \mathrm{c}$ & $2221.00 \mathrm{a}$ & $2.07 \mathrm{a}$ & $1.845 \mathrm{~b}$ & $2.29 \mathrm{a}$ & $5878.14 \mathrm{bc}$ \\
\hline \multicolumn{8}{|l|}{ Selenium } \\
\hline $\mathrm{Se} 0$ & $1505.12 \mathrm{~b}$ & $1447.28 \mathrm{~b}$ & $1600.40 \mathrm{~b}$ & $1.43 \mathrm{~b}$ & $1.59 \mathrm{~b}$ & $1.77 \mathrm{~b}$ & $6419.87 \mathrm{a}$ \\
\hline Sel & $1884.59 \mathrm{a}$ & $1728.31 \mathrm{a}$ & $1970.23 \mathrm{a}$ & $2.12 \mathrm{a}$ & $2.16 \mathrm{a}$ & $2.32 \mathrm{a}$ & $6479.82 \mathrm{a}$ \\
\hline \multicolumn{8}{|c|}{ Microelements } \\
\hline M0 & $1540.51 b$ & $1421.64 b$ & $1714.60 \mathrm{~b}$ & $1.53 \mathrm{~b}$ & $1.59 \mathrm{~b}$ & $1.94 \mathrm{~b}$ & $6618.21 \mathrm{a}$ \\
\hline M1 & $1849.20 \mathrm{a}$ & $1753.95 a$ & $1856.03 \mathrm{a}$ & $2.02 \mathrm{a}$ & $2.17 \mathrm{a}$ & $2.15 \mathrm{a}$ & $6281.47 \mathrm{~b}$ \\
\hline
\end{tabular}

Means followed by similar letters in each column are not significantly different at the $5 \%$ level of probability according to Tukey's Test. $\mathrm{L}_{1}$ : Optimum condition (control) $\mathrm{L}_{2}$ : Water limitation in $\mathrm{V}_{8}$ stage. $\mathrm{L}_{3}$ : Water limitation in blister stage. $\mathrm{L}_{4}$ : Water limitation in dough stage. Se0: Without selenium. Sel: With selenium.

M0: Without microelements. M1: With microelements. SOD: Superoxid dismutase. MAD: Malondialdehyde

Tab. 5. Mean comparison of twofold interaction effects of traits

\begin{tabular}{|c|c|c|c|c|c|c|c|c|}
\hline \multicolumn{2}{|c|}{ Treatment } & \multicolumn{3}{|c|}{ (u/mg.protein $)$} & \multicolumn{3}{|c|}{ (nm/mg.protein) } & $\begin{array}{c}\text { Grain yield } \\
\mathrm{Kg} \mathrm{ha}^{-1}\end{array}$ \\
\hline Water Limitation & Selenium & & & & & & & \\
\hline \multirow{2}{*}{ 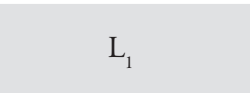 } & Se0 & $1165.00 \mathrm{f}$ & $1404.62 \mathrm{c}$ & $1449.37 d$ & $1.05 \mathrm{e}$ & $1.49 \mathrm{~d}$ & $1.79 \mathrm{e}$ & $7656.73 \mathrm{a}$ \\
\hline & Sel & $1362.87 \mathrm{e}$ & $1716.37 \mathrm{~b}$ & $1702.56 \mathrm{c}$ & $1.45 \mathrm{~d}$ & $2.00 \mathrm{bc}$ & $2.31 b$ & $6496.14 b$ \\
\hline \multirow{2}{*}{$\mathrm{L}_{2}$} & $\mathrm{Se} 0$ & $1716.68 \mathrm{c}$ & $1368.93 c$ & $1451.87 \mathrm{~d}$ & $1.70 \mathrm{c}$ & $1.49 \mathrm{~d}$ & $1.71 \mathrm{e}$ & $6616.50 \mathrm{~b}$ \\
\hline & Sel & $2345.81 \mathrm{a}$ & $1564.25 b$ & $1802.87 \mathrm{c}$ & $2.61 \mathrm{a}$ & $2.00 \mathrm{bc}$ & $2.08 \mathrm{~cd}$ & $6591.00 \mathrm{~b}$ \\
\hline \multirow{2}{*}{$\mathrm{L}_{3}$} & Se0 & $1549.50 \mathrm{~d}$ & $1672.68 b$ & $1515.37 d$ & $1.34 \mathrm{~d}$ & $1.86 \mathrm{c}$ & $1.69 \mathrm{e}$ & $6019.26 b$ \\
\hline & Sel & $1735.43 c$ & 1978.81a & $1918.50 \mathrm{~b}$ & $1.93 \mathrm{~b}$ & $2.49 \mathrm{a}$ & $2.22 \mathrm{bc}$ & $6462.83 b$ \\
\hline \multirow{2}{*}{$\mathrm{L}_{4}$} & $\mathrm{Se} 0$ & $1589.31 b$ & $1342.8 \mathrm{c}$ & $1985.0 \mathrm{~b}$ & $1.65 \mathrm{c}$ & $1.53 \mathrm{~d}$ & $1.89 \mathrm{de}$ & $5386.99 c$ \\
\hline & Sel & $2094.25 d$ & $1653.81 \mathrm{~b}$ & $2457.00 \mathrm{a}$ & $2.48 \mathrm{a}$ & $2.15 b$ & $2.69 \mathrm{a}$ & $6369.29 \mathrm{~b}$ \\
\hline Water Limitation & Microelement & & & & & & & \\
\hline \multirow{2}{*}{$\mathrm{L}_{1}$} & Mo & $1144.12 \mathrm{e}$ & $1408.12 \mathrm{c}$ & $1494.62 \mathrm{e}$ & $0.97 \mathrm{~d}$ & $1.48 \mathrm{e}$ & $1.95 \mathrm{bc}$ & $6850.14 \mathrm{ab}$ \\
\hline & M1 & $1383.75 d$ & $1712.87 \mathrm{~b}$ & $1657.31 \mathrm{~cd}$ & $1.53 \mathrm{c}$ & $2.01 \mathrm{c}$ & $2.15 b$ & $7302.73 \mathrm{a}$ \\
\hline \multirow{2}{*}{$\mathrm{L}_{2}$} & Mo & $1754.37 \mathrm{c}$ & $1311.06 \mathrm{c}$ & $1574.75 \mathrm{de}$ & $1.84 \mathrm{~b}$ & $1.56 \mathrm{e}$ & $1.82 \mathrm{c}$ & $6768.25 \mathrm{ab}$ \\
\hline & M1 & $2308.12 \mathrm{a}$ & $1622.12 b$ & $1680.00 \mathrm{~cd}$ & $2.47 \mathrm{a}$ & $1.93 \mathrm{~cd}$ & $1.97 \mathrm{bc}$ & 6439.25 bc \\
\hline \multirow{2}{*}{$\mathrm{L}_{3}$} & Mo & $1468.12 c$ & $1580.37 \mathrm{~b}$ & $1715.62 \mathrm{c}$ & $1.55 \mathrm{c}$ & $1.81 \mathrm{~d}$ & $1.92 \mathrm{bc}$ & $6422.27 \mathrm{bc}$ \\
\hline & M1 & $1636.81 \mathrm{c}$ & $2071.12 \mathrm{a}$ & $1718.25 \mathrm{c}$ & $1.72 \mathrm{~b}$ & $2.54 \mathrm{a}$ & $1.99 \mathrm{bc}$ & $6059.82 c$ \\
\hline \multirow{2}{*}{$\mathrm{L}_{4}$} & Mo & $1626.75 c$ & $1387.00 \mathrm{c}$ & $2073.43 b$ & $1.78 \mathrm{~b}$ & $1.49 \mathrm{e}$ & $2.09 \mathrm{~b}$ & $6432.19 b c$ \\
\hline & M1 & $2056.81 \mathrm{~b}$ & $1609.68 \mathrm{~b}$ & $2368.56 a$ & $2.35 \mathrm{a}$ & $2.19 \mathrm{~b}$ & $2.49 \mathrm{a}$ & $5324.09 \mathrm{~d}$ \\
\hline Selenium & Microelement & & & & & & & \\
\hline \multirow{2}{*}{$\mathrm{Se} 0$} & Mo & $1920.46 a$ & $1851.62 \mathrm{a}$ & $2041.59 \mathrm{a}$ & $2.22 \mathrm{a}$ & $2.35 \mathrm{ab}$ & $2.44 a$ & $6664.41 a$ \\
\hline & M1 & $1848.71 \mathrm{ab}$ & $1605.00 \mathrm{~b}$ & $1898.87 \mathrm{~b}$ & $2.00 \mathrm{~b}$ & $1.98 \mathrm{bc}$ & $2.20 \mathrm{~b}$ & $6175.33 b$ \\
\hline \multirow{2}{*}{ Sel } & Mo & $1777.93 \mathrm{~b}$ & $1656.28 \mathrm{~b}$ & $1670.46 c$ & $1.80 \mathrm{c}$ & $1.99 \mathrm{bc}$ & $1.86 \mathrm{c}$ & $6572.01 \mathrm{ab}$ \\
\hline & M1 & $1232.31 \mathrm{c}$ & $1238.28 \mathrm{c}$ & $1530.34 \mathrm{~d}$ & $1.07 \mathrm{~d}$ & $1.20 \mathrm{e}$ & $1.68 \mathrm{~d}$ & $6387.62 \mathrm{ab}$ \\
\hline
\end{tabular}

Means followed by similar letters in each column are not significantly different at the $5 \%$ level of probability according to Tukey's Test

malondialdehyde has been known as the end product of peroxidation of membrane lipids. Water deficit stress by the increase of generation ROS is responsible for stressdependent peroxidation of membrane lipids. It seems that the enhancement in produce ROS can increase substrate for superoxide dismutase reaction. Increased superoxide dismutase and catalase activities in response to water deficit stress have been reported (Halliwell and Gutteridge, 1989).

Our results showed that drought stress in maize farms could make the significant changes in antioxidant enzymes activity in leave, similar to those reported by Ghorbanli $e t$ 
Tab. 6. Mean comparison of threefold interaction effects of traits

\begin{tabular}{|c|c|c|c|c|c|c|c|c|c|}
\hline \multirow{2}{*}{\multicolumn{3}{|c|}{ Treatment }} & $\mathrm{SODV}_{8}$ & $\mathrm{SOD} \mathrm{R}_{2}$ & $\mathrm{SOD} \mathrm{R}_{4}$ & $\mathrm{MADV}_{8}$ & MAD R $_{2}$ & $\mathrm{MAD} \mathrm{R}_{4}$ & \\
\hline & & & \multicolumn{3}{|c|}{$(\mathrm{u} / \mathrm{mg}$ protein $)$} & \multicolumn{3}{|c|}{ (nm/mg.protein) } & $\left(\mathrm{Kg} \mathrm{ha}^{-1}\right)$ \\
\hline Water Limitation & Selenium & Microelements & & & & & & & \\
\hline $\mathrm{L}_{1}$ & $\mathrm{Se} 0$ & M0 & $1540.37 \mathrm{e}$ & $1725.37 \mathrm{~b}$ & 1781.75 ef & $1.782 \mathrm{e}$ & $2.06 \mathrm{bcd}$ & $2.41 \mathrm{~b}$ & $7336.55 \mathrm{ab}$ \\
\hline $\mathrm{L}_{1}$ & $\mathrm{Se} 0$ & M1 & $1185.37 \mathrm{~g}$ & $1707.37 \mathrm{~b}$ & $1623.37 \mathrm{fg}$ & $1.134 \mathrm{~g}$ & $1.95 \mathrm{~cd}$ & $2.20 \mathrm{bcd}$ & $7976.91 \mathrm{a}$ \\
\hline $\mathrm{L}_{1}$ & Sel & M0 & $1227.12 \mathrm{~g}$ & $1700.37 \mathrm{~b}$ & $1532.87 \mathrm{gh}$ & $1.28 \mathrm{fg}$ & $1.97 \mathrm{~cd}$ & $1.88 \mathrm{~d}-\mathrm{g}$ & $6790.73 \mathrm{bcd}$ \\
\hline $\mathrm{L}_{1}$ & Sel & M1 & $1102.87 \mathrm{~g}$ & $1108.87 \mathrm{c}$ & $1365.87 \mathrm{~h}$ & $0.81 \mathrm{~h}$ & $1.01 \mathrm{f}$ & $1.69 \mathrm{efg}$ & $6628.55 \mathrm{bc}$ \\
\hline $\mathrm{L}_{2}$ & $\mathrm{Se} 0$ & M0 & $2458.62 \mathrm{a}$ & $1625.00 \mathrm{~b}$ & 1834.50 de & $2.73 \mathrm{a}$ & $2.02 \mathrm{~cd}$ & $2.18 \mathrm{bcd}$ & $6799.52 \mathrm{bc}$ \\
\hline $\mathrm{L}_{2}$ & $\mathrm{Se} 0$ & M1 & $2233.00 \mathrm{~b}$ & $1505.50 \mathrm{~b}$ & $1771.25 \mathrm{ef}$ & $2.48 \mathrm{ab}$ & $1.98 \mathrm{~cd}$ & $1.97 \mathrm{c}-\mathrm{f}$ & $6433.48 \mathrm{bcd}$ \\
\hline $\mathrm{L}_{2}$ & Sel & M0 & $2157.62 b c$ & $1621.25 \mathrm{~b}$ & $1525.50 \mathrm{gh}$ & $2.21 \mathrm{~cd}$ & $1.84 \mathrm{de}$ & $1.76 \mathrm{efg}$ & $6736.97 \mathrm{bc}$ \\
\hline $\mathrm{L}_{2}$ & Sel & M1 & $1275.75 \mathrm{fg}$ & $1116.62 \mathrm{c}$ & $1387.52 \mathrm{~h}$ & $1.20 \mathrm{~g}$ & $1.14 \mathrm{f}$ & $1.67 \mathrm{fg}$ & $6445.03 \mathrm{bcd}$ \\
\hline $\mathrm{L}_{3}$ & $\mathrm{Se} 0$ & M0 & $1453.25 \mathrm{ef}$ & $2398.25 \mathrm{a}$ & $1884.00 \mathrm{de}$ & $1.93 \mathrm{e}$ & $3.03 \mathrm{a}$ & $2.22 b c$ & $6448.16 \mathrm{bcd}$ \\
\hline $\mathrm{L}_{3}$ & $\mathrm{Se} 0$ & M1 & $2017.62 \mathrm{~cd}$ & $1559.37 \mathrm{~b}$ & $1953.00 \mathrm{~cd}$ & $1.93 \mathrm{e}$ & $1.96 \mathrm{~cd}$ & $2.21 b c$ & $5590.36 \mathrm{~d}$ \\
\hline $\mathrm{L}_{3}$ & Sel & M0 & $1843.00 \mathrm{~d}$ & $1744.00 \mathrm{~b}$ & $1552.50 \mathrm{~g}$ & $1.36 f$ & $2.06 \mathrm{bcd}$ & $2.76 \mathrm{efg}$ & $6396.38 \mathrm{bcd}$ \\
\hline $\mathrm{L}_{3}$ & Sel & M1 & $1256.00 \mathrm{~g}$ & $1601.37 \mathrm{~b}$ & $1478.25 \mathrm{gh}$ & $1.17 \mathrm{~g}$ & $1.67 \mathrm{e}$ & $1.62 \mathrm{~g}$ & $6529.28 \mathrm{bcd}$ \\
\hline $\mathrm{L}_{4}$ & $\mathrm{Se} 0$ & M0 & $2229.62 \mathrm{~b}$ & $1659.87 \mathrm{~b}$ & $2666.12 \mathrm{a}$ & $2.52 \mathrm{ab}$ & $2.27 \mathrm{~b}$ & $2.96 \mathrm{a}$ & $6073.43 \mathrm{~cd}$ \\
\hline $\mathrm{L}_{4}^{4}$ & $\mathrm{Se} 0$ & M1 & $1958.87 \mathrm{~d}$ & $1647.75 \mathrm{~b}$ & $2247.87 \mathrm{~b}$ & $2.44 b c$ & $2.02 \mathrm{~cd}$ & $2.42 \mathrm{~b}$ & $4700.55 \mathrm{e}$ \\
\hline $\mathrm{L}_{4}$ & Sel & M0 & $1884.00 \mathrm{~d}$ & $1559.50 \mathrm{~b}$ & $2071.00 \mathrm{c}$ & $2.19 \mathrm{~d}$ & $2.10 \mathrm{bc}$ & $2.02 \mathrm{cde}$ & 6363.96bcd \\
\hline $\mathrm{L}_{4}^{4}$ & Sel & M1 & $1294.62 \mathrm{fg}$ & $1126.25 \mathrm{c}$ & $1899.00 \mathrm{de}$ & $1.11 \mathrm{~g}$ & $0.97 \mathrm{f}$ & $1.76 \mathrm{fg}$ & $5947.62 \mathrm{~cd}$ \\
\hline
\end{tabular}

Means followed by similar letters in each column are not significantly different at the $5 \%$ level of probability according to Tukey's Test

al. (2004), Malan et al. (1990), Bailly et al. (2000), Giang and Huang (2001) and Habibi et al. (2004) in sunflower.

Selenium and microelement fertilizers could also increase superoxide dismutase enzyme activity and malondialdehyde content in leaves in $\mathrm{V}_{8}, \mathrm{R}_{2}$ and $\mathrm{R}_{4}$ stages. Selenium role in plants under water stress could increase antioxidant enzymes activities by reducing oxidative conditions and free radicals which have a determinate effect on plant cells.

According to means comparison microelements application was increased antioxidant activities of superoxide dismotase in $\mathrm{V}_{8}, \mathrm{R}_{2}$ and $\mathrm{R}_{4}$ plant growth and development stages by $16.7 \%, 18.9 \%$ and $7.6 \%$. The malondialdehyde content of leaves was increased by $24 \%, 26.7 \%$ and $9.7 \%$ respectively compared to control (Tab. 4). Rahimizade et al. (2007) reported that SOD activity was increased by $31 \%$, under drought stress in sunflower. Hacisalihoglu et al. (2003) reported that under $Z n$ deficiency stress, activity of $\mathrm{Cu} / \mathrm{Zn} \mathrm{SOD}$ decreases as $\mathrm{Zn}$ is directly involved in both gene expression and protein synthesis. Cakmak (2000) reported that $\mathrm{Zn}$ deficiency stress may inhibit the activities of a number of antioxidant enzyme. Similarly, Rahmati $e t$ al. (2004) found that the activity of SOD, CAT and APX (ascorbate peroxidase) in excess $\mathrm{Mn}$ treated cells increased compared to control treatment.

Using selenium in water deficit stress condition increased superoxide dismutase enzyme activity and malondialdehyde content as compared to treatments of not using selenium. The highest amounts of measured traits under water deficit were found in $\mathrm{V}_{8}$ and $\mathrm{R}_{4}$ stages (Tab. 5).

The effects of three way factors interactions on superoxide dismutase enzyme activity and malondialdehyde $\mathrm{V}_{8}$ and $R_{2}$ stages were significant but, in $R_{4}$ stages not significant. In water deficit stress condition in each three stage, the highest malondialdehyde content were observed from treatments of without microelements and without selenium. This might indicate plant sensitivity due to no protection factor under water stress.

It was found in this study that superoxide dismutase enzyme had positive and significant correlation with malondialdehyde content under water deficit stress in vegetative stage $\left(V_{8}\right)$, in blister stage $\left(R_{2}\right)$ and water deficit stress in dough stage $\left(\mathrm{R}_{4}\right)$ (Tab. 7).

Tab. 7. Matrix of simple correlation coefficient among different traits

\begin{tabular}{cccccccc}
\hline & SODV $_{8}$ & MADV $_{8}$ & SODR $_{2}$ & MADR $_{2}$ & SODR $_{4}$ & MADR $_{4}$ & Grain yield \\
\hline SODV $_{8}$ & 1 & & & & & & \\
MADV $_{8}$ & $0.92^{* *}$ & 1 & & & & & \\
SODR $_{2}$ & $0.34^{*}$ & $0.46^{* *}$ & 1 & & & & \\
MADR $_{2}$ & $0.61^{* *}$ & $0.71^{* *}$ & $0.83^{* *}$ & 1 & & & \\
SODR $_{4}$ & $0.65^{* *}$ & $0.69^{* *}$ & $0.36^{* *}$ & $0.56^{* *}$ & 1 & $-0.10^{\text {ns }}$ & 1 \\
Grain yield $^{*}$ & $-0.12^{\text {ns }}$ & $-0.18^{*}$ & $-0.17^{*}$ & $-0.18^{*}$ & $-0.06^{\text {ns }}$ & & \\
\hline
\end{tabular}

**, *, ns significant at the $1 \%, 5 \%$ probability levels and non significant respectively 
158

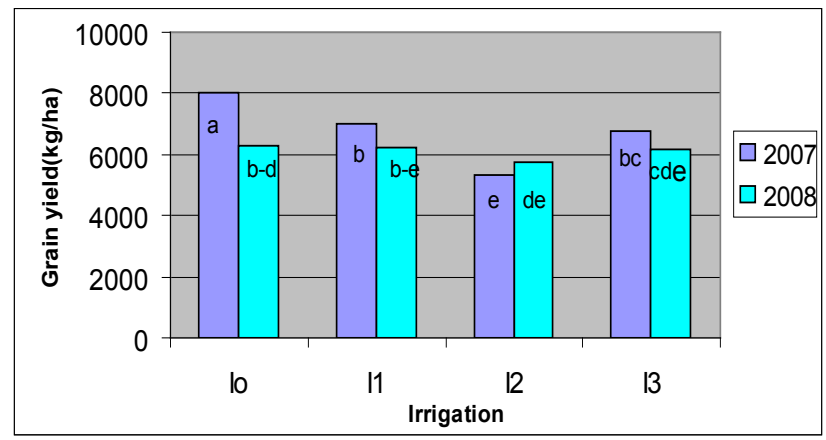

Fig. 3. Effect irrigation and year on grain yield

The result showed that grain yield was higher in the first year than the second year (Tab. 4 and Fig. 3). This seems to be related to better climate condition in the first year (Tab. 1). The effect of water deficit stress on grain yield was significant. The highest grain yield was obtained from normal irrigation and water deficit at $\mathrm{V}_{8}$ stage without significant difference (Tab. 4). It seems that under drought stress in maize the antioxidative defence system enhances sugar and starch accumulation in cells.

Grain yield of selenium sprayed-plants was increased as compared with control (Tab. 4). It may be due to enhancement of photosynthesis and decrease in leaf senescence, increases assimilate production and transport towards seeds and as a result seed yield (Xue and Hartikaine 2001).

Grain yield of microelements sprayed-plants was decreased by $5 \%$ as compared to no microelements application. This may be related to antagonistic interaction of microelements with each other. These results are similar to those reported by Himayatullah and Khan (1998). They reported that in maize, copper application alone and with iron and manganese decreased kernel number per ear, 1000 grain weight and grain yield.

Results of combined analysis two way interactions of water deficit stress and selenium on grain yield was significant. The highest grain yield was obtained from control treatment (without stress and without selenium) which showed significant differences compared to other treatments. Least grain yield under water deficit was obtained from treatment of water deficit stress in grain filling stage and without selenium application (Tab. 5). This might indicate plant sensitivity due to the lack of a protection factor under water stress. Xue et al. (2001) reported that selenium has antioxidant properties and under conditions of environmental stress, especially water stress, it can scavenge reactive oxygen. Seppanen et al. (2003) reported that selenium prevents chlorophyll degradation under water stress.

Microelement applications under optimum irrigation increased grain yield compared to non microelement application. But in water deficit stress conditions, grain yield was decreased in all growth and development stages. It seems that, in water deficit conditions due to disorder transmission and increase concentration of microelements plant toxicity was created.

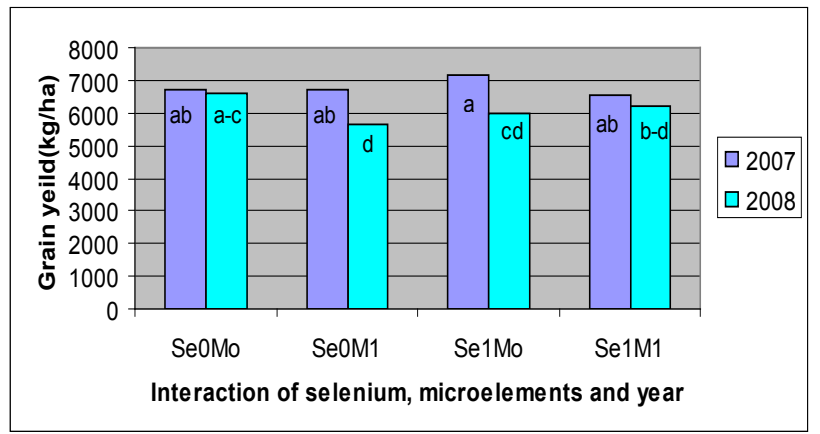

Fig. 4. Effect irrigation, selenium and year on grain yield

An antagonistic interaction was found for selenium and microelements application for grain yield. This may be due to antagonistic interaction between microelements and selenium (Tab. 5 and Fig. 4). Interactions effect had also a significant result on grain yield. The highest grain yield up to $9519 \mathrm{kgha}^{-1}$ was obtained from optimum irrigation conditions, no selenium and microelements application treatment (Tab. 6). Selenium and microelements application in vegetative growth stage and dough grain development stages in water deficit conditions could increase maize grain yield significantly (Tab. 6). It seems that in the end of the growth and development stage in increased water stress conditions, selenium and microelements find biological activity roles in plant cells. Plant tissue health and protection of cell membranes were formed as a permanent function. In general, by selenium and microelements application under water stress, higher grain yield was obtained. These study results also showed that there is a negative and significant correlation between grain yield with superoxide dismutase enzyme and malondialdehyde content under water deficit in the vegetative stage, blister stage and water deficit in dough grain development stages (Tab. 7). This was observed as the decrease of grain yield.

\section{Conclusions}

Microelements and selenium application on maize farms could not only increased superoxide dismutase enzyme activity and malondialdehyde content in leaf tissues under normal irrigation but also, under water deficit in $\mathrm{V}_{8}$, $\mathrm{R}_{2}$ and $\mathrm{R}_{4}$ stages. Grain yield by selenium application was increased. The role of selenium in plants under stress was to increase antioxidant enzyme activities to reduce oxidative conditions or free radical injures which have a determinate effect on plant cells. The effect of microelements acts as a component of superoxide dismutase, catalase, peroxidase and nitrate reductase. Therefore, when plants are deficient in these elements, activities of antioxidant enzymes decrease, thus imposing and increased sensitivity to environmental stresses. Results showed that with microelements in water optimum conditions, and selenium spray under water deficit stress condition an optimum grain yield can be obtained. 


\section{References}

Alizadeh A (2002). Designing of irrigation system. Ferdowsi university, Mashhad, Iran, 655p.

Alscher RG, Erturk N, Heath LS (2002). Role of superoxide dismutase in controlling oxidative stress in plants. J Exp Bot 53(327):1331-1341.

Bailly C, Benamar A, Corbineau F, Come D (2000). Antioxidant systems in sunflower seeds as affected by priming. Seed Sci Res 10(2):35-42.

Cakmak I (2000). Possible roles of zinc in protecting plant cells from damage by reactive oxygen species. New Phytol 146(2):85-200.

Cheong YH, Kim KN, Pandey GK, Gupta R, Grant JJ, Luan S (2003). CLB1, a calcium sensor that differentially regulates salt, drought, and cold responses in Arabidopsis. Plant Cell 15:1833-1845.

Cushman JC, Bohnert HJ (2000). Genomic approaches to plant stress tolerance. Curr Opin Plant Biol 3:117-24.

Elstner EF (1991). Mechanism of oxygen activation in different compartments of plant cell, 13-25 p. In: Pell EJ, Steffen KL (Eds.). Active Oxygen/Oxidative Stress and Plant Metabolism. Amer Soc Plant Physiology, Rockville.

Farshi AA, Sharyati MR, Jarallahi R, Ghaemi MR, Shahabifar M, Toulaie MM (1997). Estimate of major crops and garden water requerment in iran. Issuance Education of Agriculture, $900 \mathrm{p}$.

Ghorbanli M, Ebrahimzadeh H, Sharifi M (2004). Effects of $\mathrm{NaCl}$ and mycorrhizal fungi on antioxidative enzymes in soybean. Biol Plant 48:575-581.

Giang Y, Huang B (2001). Drought and heat stress injury two Cool-Season Turf grasses in relation to antioxidant metabolism and lipid Peroxidation. Crop Sci 41:436-442.

Habibi D, Boojar MM, Mahmoudi A, Ardakani MR, Taleghani D (2004). Antioxidative enzymes in sunflower subjected to drought stress. $4^{\text {th }}$ International Crop Science Congress, Australia.

Hacisalihoglu G, Hart JJ, Wang YH, Cakmak I, Kochian LV (2003). Zinc efficiency is correlated with enhanced expression and activity of zinc-requiring enzymes in wheat. Plant Physiol 131:595-602.

Hagar H, Ueda N, Shal SV (1996). Role of reactive oxygen metabolites in DNA damage and cell death in chemical hypoxic injury LLC-PK1 cells. Amer J Physiol 271:209215.

Halliwell B, Gutteridge JMC (1989). Free Radicals in Biology and Medicine. Oxford, Clarendon Press.

Himayatullah B, Kan M (1998). Response of irrigated maize to trace elements in the presence of NPK sarhad. J Agric 14:117-120.

Madhava Rao KV, Sresty TVS (2000). Antioxidative parameters in the seedlings of pigeonpea (Cajanus cajan L. Millspaugh) in response to $\mathrm{Zn}$ and Ni stresses. Plant Sci 157:113-128.
Malakuti MG, Sepehri A (2001). Nutrition of optimum oil seeds method of effective at received to selfing of oil in Iran. KHaniran Thehran Press.

Malan C, Greylingand MM, Gressel J (1990). Correlation between $\mathrm{Cu}$. Zn superoxide dismutase and xenobiotic stress tolerance in maize inbreds. Plant Sci 69:157-166.

Misra HP, Fridorich I (1979). The generation of superoxide radical during photoxidation. J Biol Chem 247:6960-6966.

Morris ML, Risopoulos J, Beck D (1999). Genetic Changes in Farmer- recycled Maize Seed: A Review of the Evidence. CIMMYT Econ. Working Paper No. 99-07. Mexico, D.F., CIMMYT.

Movahed Dehnavi M, Modarres Sanavi AM, Soroushzadae A, Jalali M (2002). Effect foliar application of zinc and manganese on yield and yield components of three safflower cultivars under drought stress in Isfahan. Abstract of articles $8^{\text {th }}$ Congress of Agronomy and Plant Breeding. Rasht. Iran.

Paylore P, Greenwell JR (1979). Fools run in pinpointing the arid zones. J Arid Environ 10:17-18.

Rahmati M, Yazdani M, Ghanati F (2004). Effect of excess amount of $\mathrm{Mn}$ on activation of certain enzymes of antioxidant system in suspension-cultured tea cells. The 2nd Congress on Applied Biology (International Approach). 2930 Sep. 2004, Mashhad, Iran.

Rahimizade M, Habibi D, Madani H, Mohammadi GN, Mehraban A, Sabet AM (2007). Effects of microelements on Antioxidat enzymes metabolism in sunflower Under drought stress . Hella 30(47):167-174.

SAS Institute Inc (1997). SAS User's Guide. Statistical Analysis Institiute Inc.

Scandalios JG (1997). Molecular Genetics of Superoxide Dismutases in Plants, 527-568 p. In: Scandalios JG (Ed.). Oxidative Stress and the Molecular Biology of Antioxidative Defenses. Plainview, Cold Spring Harbor.

Seppanen M, Turakainen M, Hartikainen H (2003). The effect of selenium on photooxidative stress tolerance in potato. Plant Sci 165:311-319.

Upadhyaya H, Panda SK (2004). Responses of Camellia senensis to drought and rehydration. Biol Plant 48:597-600.

Wang SH, Yang ZM, Yang H, Lu B, Li SQ, Lu YP (2004). Copper-induced stress and antioxidative responses in roots of Brassica juncea. Bot Bull Academia Sinica 45:203-212.

Xiong L, Schumaker KS, Zhu JK (2002). Cell signaling during cold, drought, and salt stress. Plant Cell 14:165-183.

Xue T, Hartikainen H, Piironen V (2001). Antioxidative and growth-promoting effect of selenium in senescing lettuce. Plant and Soil 27:55-61.

Yao X, Cho J, Wang G (2009). Effects of selenium on wheat seedlings under drought stress. Biol Trace Elem 130:283290. 